\title{
Relationship between age and surgical success after trabeculectomy with adjunctive mitomycin C
}

\author{
Thi Kieu Hau Hoang ${ }^{1,2} \cdot$ Young Kook Kim ${ }^{1} \cdot$ Jin Wook Jeoung ${ }^{1} \cdot$ Ki Ho Park $\mathbb{D}^{1}$
}

Received: 26 May 2017 / Revised: 11 January 2018 / Accepted: 31 January 2018 / Published online: 27 March 2018

(C) The Royal College of Ophthalmologists 2018

\begin{abstract}
Purpose To investigate whether there is any effect of age on bleb morphology and surgical success after trabeculectomy with adjunctive Mitomycin C (MMC).

Methods A total 143 eyes of 123 patients were divided into two groups: group 1 ( $\leq 60$ years old, $n=85$ ) and group 2 ( $>60$ years old, $n=58$ ). Kaplan-Meier survival curve analysis was used to assess long-term surgical success according to "complete success" (intraocular pressure (IOP) reduction $\geq 30 \%$ without medications) and "qualified success" criteria (IOP reduction $\geq 30 \%$, with or without medications) between the two groups. Cox proportional hazard models were used to examine the factors associated with surgical success. Bleb morphology was evaluated by height, extent and vascularity at postoperative 1 month, 3 months, 6 months, and 12 months.

Results The younger-age group (group 1) showed significantly better results, in terms of both complete success and qualified success, than did the older-age group (group 2) $(p<0.001$ and $p=0.01$, respectively). Age and preoperative anti-glaucoma medications were the factors related to complete success $(p=0.001)$. Also, there were significant differences in height, extent and vascularity of bleb between the two groups $(p=0.038, p=0.002$ and $p=0.006$, respectively).

Conclusion The long-term surgical outcome after trabeculectomy with MMC was better in the younger-age group than in the older-age group. Specifically, the younger-age group showed a more prominent and more localized bleb with less vascularity.
\end{abstract}

\section{Introduction}

Trabeculectomy was first introduced as a surgical technique for glaucoma by Cairns in 1968 [1]; since that time, it has been the favored procedure for surgical management of various types of glaucoma. The purpose of trabeculectomy is to reduce intraocular pressure (IOP) in order to avoid progression of optic nerve damage. Success is dependent

Electronic supplementary material The online version of this article (https://doi.org/10.1038/s41433-018-0071-x) contains supplementary material, which is available to authorized users.

Ki Ho Park

kihopark@snu.ac.kr

1 Department of Ophthalmology, Seoul National University Hospital, Seoul, Korea

2 Department of Ophthalmology, Binh Duong General Hospital, Hanoi, Vietnam not only on preoperative and intraoperative conditions but also on the persistence of filtration efficiency.

Trabeculectomy success is associated largely with age, as wound healing typically slows as people grow older [2]. As a means of improving the probability of trabeculectomy success, Mitomycin C (MMC) is commonly administered [3-5]. However, to our knowledge, no study has compared the long-term success rate and bleb morphology after trabeculectomy with adjunctive MMC between different age groups. In this study, we endeavored to determine whether there is any effect of age on success rate and bleb morphology after trabeculectomy with adjunctive MMC.

\section{Materials and methods}

\section{Patient selection}

A retrospective review was conducted of patients who had undergone trabeculectomy with adjunctive MMC as a first glaucoma filtration surgery at the Department of 

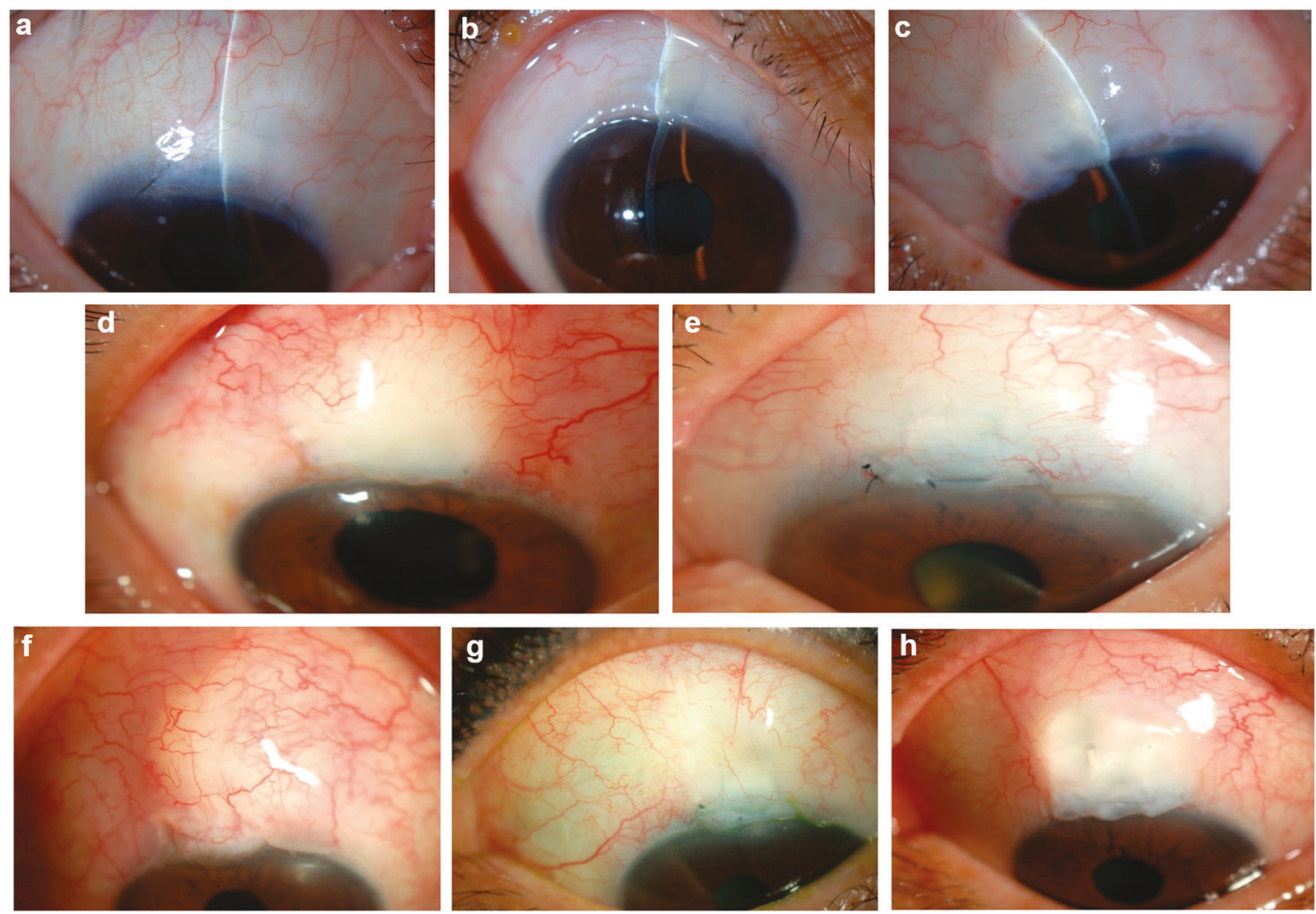

Fig. 1 Grading of bleb height: a H0, elevated flat bleb, b H1, bleb height $\leq 1 \mathrm{~mm}$, and $\mathbf{c} \mathrm{H} 2$, bleb height $>1 \mathrm{~mm}$. Grading of bleb extent: d E0, $\leq 3$ clock hours, and e E1,>3 clock hours. Grading of bleb vascularity: f V0, normal vascularity, g V1, hypovascularity, and h V2, avascularity

Ophthalmology, Seoul National University Hospital, between January 2007 and December 2015 and been followed up on for at least 12 months postoperatively. The exclusion criterion was any additional glaucoma filtration surgery performed after the first trabeculectomy. Both eyes were included if both met the inclusion criteria. A total 143 eyes of 123 patients were enrolled and subsequently divided into two age groups: group 1, patients 60 years of age or younger at surgery, and group 2, patients older than 60 years at surgery. The baseline variables were age at surgery, gender, type of glaucoma, presence of diabetes mellitus or systemic hypertension, family history of glaucoma, preoperative IOP, number of preoperative anti-glaucoma medications, preoperative and postoperative cataract surgery, and visual field index (mean deviation).

\section{Surgical technique}

The trabeculectomy was performed by a single surgeon (KHP) applying the same surgical procedures and using the same MMC concentration $(0.4 \mathrm{mg} / \mathrm{ml})$ and duration $(2 \mathrm{~min})$ in all cases. After topical anesthesia with proparacaine $0.5 \%$, the eye was prepared and draped. A corneal traction suture was placed with 6-0 silk, and a fornix-based conjunctival flap was formed at the superior limbus. After dissection of a $3 \times 3 \mathrm{~mm}^{2}$ rectangular scleral flap with half thickness, MMC was applied by 4-5 blocks of $2 \times 2 \mathrm{~mm}$ sized cellulose sponges placed on the episclera over 2 clock hours at a concentration of $0.4 \mathrm{mg} / \mathrm{ml}$ for $2 \mathrm{~min}$. Thereafter, MMC was washed with $10 \mathrm{ml}$ of balanced salt solution. A full-thickness ostium was excised at the trabecular meshwork with a punch, and an iridectomy was performed. Upon completion, the scleral flap was sutured with 10-0 nylon at each corner. In addition, two limbal sutures at the edge of the conjunctival flap and one mattress suture in the middle of the limbal wound were applied with 10-0 nylon. Finally, a subconjunctival dexamethasone injection was performed.

\section{Criteria of surgical success}

The surgical outcome was judged based on the IOPlowering effect during the follow-up period. Surgical success was classified as either complete success and qualified success. Complete success was defined as follows: reduction of postoperative IOP by 30 or more percent of preoperative IOP without anti-glaucoma medication or any additional intervention (bleb needling with or without 5fluorouracil and/or any additional glaucoma surgery) or postoperative hypotony. Qualified success was defined as follows: reduction of postoperative IOP by 30 or more percent of preoperative IOP with or without anti-glaucoma medication or any additional intervention or postoperative 
Table 1 Baseline characteristics of patients in group $1($ age $\leq 60)$ and group 2 (age $>60$ )

\begin{tabular}{llll}
\hline Characteristic & Group 1 & Group 2 & $P$-value \\
\hline Number of eyes & 85 & 58 & \\
Male/Female & $45 / 40$ & $37 / 21$ & $0.23^{b}$ \\
Right/Left eye & $46 / 39$ & $27 / 31$ & $0.40^{b}$ \\
Family history of glaucoma & 3 & 0 & $0.27^{b}$ \\
Diabetes mellitus & 15 & 17 & $0.11^{b}$ \\
Hypertension & 16 & 21 & $0.02^{b}$ \\
Type of glaucoma & & & $0.03^{a}$ \\
POAG & $43(50.6 \%)$ & $41(70.7 \%)$ & \\
PACG & $3(3.5 \%)$ & $3(5.2 \%)$ & \\
UG & $19(22.4 \%)$ & $7(12.1 \%)$ & \\
DG & $9(10.6 \%)$ & $0(0 \%)$ & \\
OSG & $11(12.9 \%)$ & $7(12.1 \%)$ & \\
Previous cataract surgery & 7 & 7 & $0.57^{\mathrm{b}}$ \\
Phacotrabeculectomy & 3 & 6 & $0.10^{\mathrm{b}}$ \\
Cataract surgery after TLE & 11 & 18 & $0.01^{\mathrm{b}}$ \\
No. of anti-glaucoma & $4.1 \pm 0.7$ & $4.0 \pm 0.9$ & $0.89^{\mathrm{a}}$ \\
medications & & & \\
Preoperative IOP (mmHg) & $29.7 \pm 7.5$ & $26.5 \pm 6.2$ & $0.01^{\mathrm{a}}$ \\
Postoperative IOP (mmHg) & $13.5 \pm 3.8$ & $13.7 \pm 2.9$ & $0.69^{\mathrm{a}}$ \\
Visual field index (MD) & $-13.9 \pm$ & $-15.9 \pm 8.9$ & $0.30^{\mathrm{a}}$ \\
& 10.1 & & \\
\hline
\end{tabular}

TLE trabeculectomy, postoperative IOP mean IOP after trabeculectomy during the follow-up time, $P O A G$ Primary open-angle glaucoma, $P A C G$ Primary angle-closure glaucoma, $U G$ Uveitic glaucoma, $D G$ Developmental glaucoma, $O S G$ Other secondary glaucoma, $M D$ mean deviation of visual field indexes before trabeculectomy.

a Student's $t$-test

${ }^{\mathrm{b}}$ Fisher's exact test

${ }^{c}$ number of preoperative anti-glaucoma medications

hypotony. Preoperative IOP was measured on the day before surgery. Postoperative hypotony was defined as follows: IOP less than or equal to $5 \mathrm{mmHg}$ for more than 2 months after trabeculectomy. The definition of IOP reduction should be met on two consecutive visits. A requirement for bleb massage was not considered as a failure, as it is one of the routine postoperative procedures to facilitate filtering. Laser suture lysis was allowed in all cases for both complete and qualified success criteria. The postoperative IOP and bleb morphology were assessed at 1 month, 3 months, 6 months, 12 months, 18 months, and 24 months, and annually thereafter.

\section{Grading of bleb morphology}

The filtration bleb was assessed under slit lamp by a single glaucomatologist (Park KH) and characterized with respect to height, extent and vascularity
1. Bleb height was graded according to the vertical dimension (the extent of elevation of the conjunctival flap above the scleral surface): H0, flat bleb without visible elevation; $\mathrm{H} 1$, moderately elevated bleb of a height of $1 \mathrm{~mm}$ or less; $\mathrm{H} 2$, prominent bleb of a height of more than $1 \mathrm{~mm}$ (Fig. 1a-c)).

2. Bleb extent was graded according to the horizontal dimension and based on clock hours: E0, localized bleb of extent equal to three clock hours or less; E1, diffuse bleb of extent greater than three clock hours (Fig. 1d, e)).

3. Bleb vascularity was graded according to vessel visibility on the conjunctiva over the bleb site: V0, normal vascularity (the same as that of the adjacent healthy conjunctiva); V1, hypovascularity (less vascularity on the conjunctiva over the bleb compared with the healthy conjunctiva); V2, avascularity (white, transparent or opaque bleb, without any blood vessels) (Fig. 1f-h)).

\section{Data analysis}

The data collected were entered into Excel spreadsheets and analyzed using SPSS version 22.0 (SPSS, Chicago, IL, USA) and Excel for Windows. The correlation between two numeric variables was calculated, and an analysis of variance was performed to assess the difference in mean values between the two age groups. The significance of association and differences among the categorical variables were assessed by Chi-square test and Student's $t$ test for independent samples/Wilcoxon rank sum test, respectively. The differences in bleb morphology between the two groups were tested using the Chi-square test. Surgical success was assessed by Kaplan-Meier survival curve analysis, with the $\mathrm{p}$ value derived from a log-rank test. A $P$ value less than 0.05 was considered significant. Cox proportional hazard models were used to examine the association between the patients' baseline characteristics and the trabeculectomy success rate. The variables of interest possibly affecting surgical success (i.e., those associated with success at the $P \leq 0.10$ level) were entered into univariate hazard models. In our study, variables were tested in univariate models initially, and those with $\mathrm{P}<10 \%$ were selected for the multivariate models. Then, a multivariate model for every criteria of surgical success with a set of explanatory variables was run, and each was independently and significantly associated with success at a level of $P<0.05$.

\section{Results}

\section{Baseline characteristics}

One hundred and forty-three (143) trabeculectomies with adjunctive MMC as performed in 143 eyes of 123 patients 

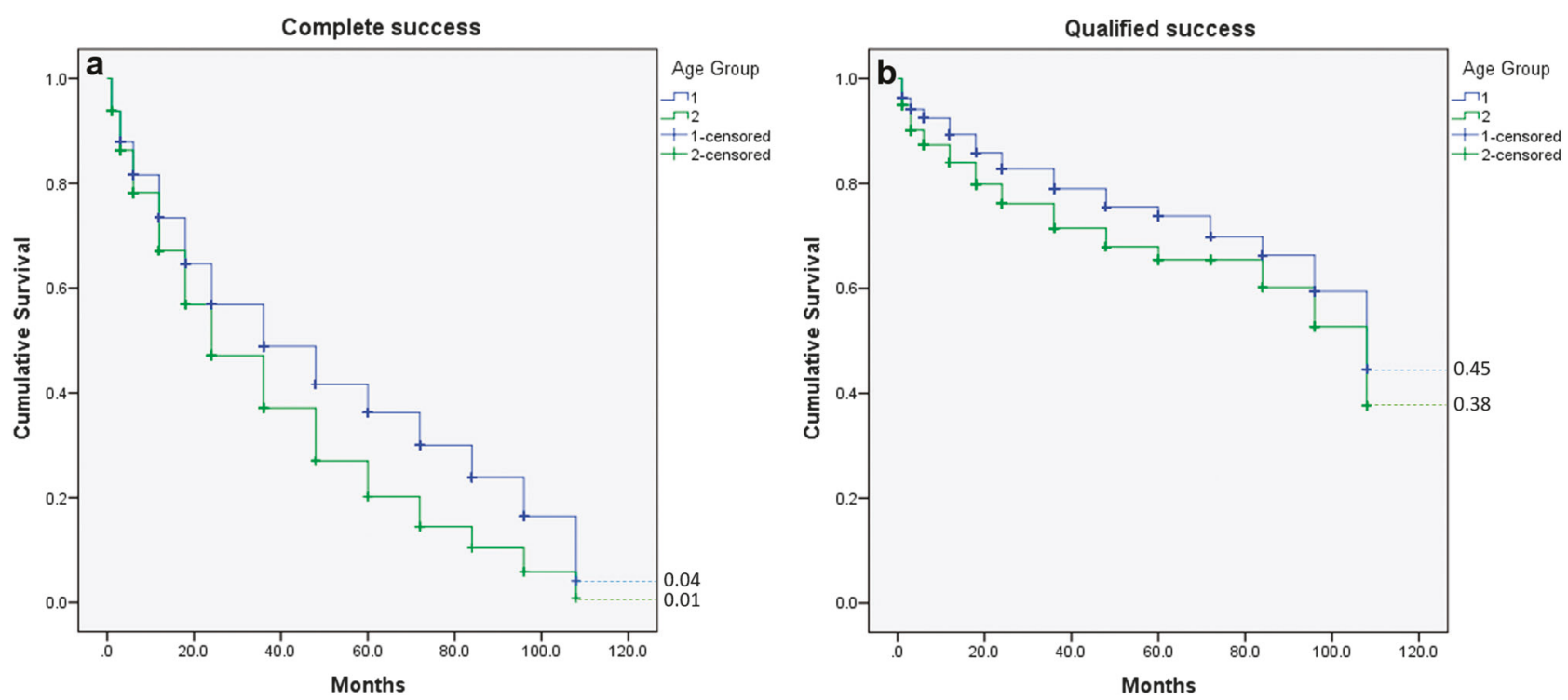

Fig. 2 Cumulative-survival-curve-determined percentages of eyes with a complete success (IOP reduction $\geq 30 \%$ without medications) and $\mathbf{b}$ qualified success (IOP reduction $\geq 30 \%$, with or without medications)

fulfilled the inclusion criteria. The mean age of the patients at surgery was $50 \pm 19.7$ years (range: $4-78$ ): $37.8 \pm 16.4$ years (range: 4-60) in group 1, and $68.1 \pm 4.5$ (range: 61-78) in group 2. The baseline characteristics are summarized in Table 1. No significant differences were observed between the two groups in gender, family history of glaucoma, the presence of diabetes mellitus, previous cataract surgery or visual field index. The commonest type of glaucoma in both groups was primary open-angle glaucoma (POAG), though there were significant inter-group prevalence differences for each of the five glaucoma types $(P=0.03)$. There was also a significant inter-group difference in the presence of hypertension $(P=0.02)$. The mean number of preoperative anti-glaucoma medications showed no significant difference between the groups: $4.1 \pm 0.7$ and $4.0 \pm 0.9$, respectively $(P=0.89)$. The preoperative IOP in both groups $(29.7 \pm 7.5$ and $26.5 \pm 6.2)$ was significant reduced, to $13.5 \pm 3.7$ and $13.7 \pm 2.9$, respectively, posttrabeculectomy $(P<0.001)$. The mean reduction of IOP was $53.4 \pm 15.6$ and $46.5 \pm 13.9 \%$, respectively $(P<0.001)$.

\section{Survival analysis}

Surgical success (complete success and qualified success) was determined by Kaplan-Meier survival curve analysis. The cumulative results are plotted in Fig. 2. The median length of follow-up from the day of surgery to the most recent visit was 48 months (4 years), and the mean was $54.6 \pm 1.1$ months. As the plots indicate, there were highly significant differences in complete success and qualified success between the two age groups $(P<0.001$ and $P=0.01$, respectively).
A total of nine eyes showed leakage from the wound within 3 weeks after surgery (5 eyes in group 1, 4 eyes in group 2). Postoperative hypotony (IOP $\leq 5 \mathrm{mmHg}$ for $>2$ months after trabeculectomy) was observed in one eye in the younger-age group. None of the eyes developed late bleb leakage, bleb infection, or postoperative endophthalmitis.

\section{Bleb morphology}

All of the patients were assessed for bleb morphology from 1 month to 12 months postoperatively. Table 2 shows the two age groups' bleb parameters at 1 month, 3 months, 6 months, and 12 months postoperatively. There was no significant difference in any of the bleb parameters at 1 month and 3 months, whereas there were significant differences in vascularity of bleb and bleb extent between the groups at 6 months $(P=0.004)$ and 12 months $(P=0.006$ and $P=0.002$, respectively). The bleb height showed a significant difference at 12 months $(P=0.04)$. The younger-age group showed a more prominent and more localized bleb with less vascularity (Supplementary Figure 1).

\section{Risk factors related to success of surgery}

\section{Complete-success criteria}

Univariate-analysis factors associated with complete success and qualified success $(P \leq 0.10)$ were age at surgery $(P<0.001)$, family history of glaucoma $(P=0.03)$, the presence of systemic hypertension $(P=0.03)$, type of 
Table 2 Parameters of bleb morphology in two groups during follow-up time after trabeculectomy with MMC

\begin{tabular}{|c|c|c|c|c|c|c|c|c|}
\hline \multirow[b]{2}{*}{ Characteristic } & \multicolumn{2}{|l|}{1 month } & \multicolumn{2}{|l|}{3 months } & \multicolumn{2}{|l|}{6 months } & \multicolumn{2}{|l|}{12 months } \\
\hline & Group $1^{\mathrm{b}}$ & Group $2^{b}$ & Group $1^{\mathrm{b}}$ & Group $2^{\mathrm{b}}$ & Group $1^{\mathrm{b}}$ & Group $2^{b}$ & Group $1^{\mathrm{b}}$ & Group $2^{b}$ \\
\hline \multicolumn{9}{|l|}{ Height } \\
\hline $\mathrm{HO}$ & 7.1 & 3.4 & 1.2 & 1.7 & 1.2 & 1.7 & 1.2 & 3.4 \\
\hline $\mathrm{H} 1$ & 23.5 & 25.9 & 21.2 & 29.3 & 18.8 & 32.8 & 17.6 & 34.5 \\
\hline $\mathrm{H} 2$ & 69.4 & 70.7 & 77.6 & 69 & 80 & 65.5 & 81.2 & 62.1 \\
\hline$P$ value $^{\mathrm{a}}$ & 0.64 & & 0.51 & & 0.15 & & 0.04 & \\
\hline \multicolumn{9}{|l|}{ Extent } \\
\hline E0 & 58.8 & 55.2 & 64.7 & 51.7 & 70.6 & 46.6 & 69.4 & 43.1 \\
\hline E1 & 41.2 & 44.8 & 35.3 & 48.3 & 29.4 & 53.4 & 30.6 & 56.9 \\
\hline$P$ value $^{\mathrm{a}}$ & 0.67 & & 0.12 & & 0.004 & & 0.002 & \\
\hline \multicolumn{9}{|l|}{ Vascularity } \\
\hline V0 & 55.3 & 69 & 42.4 & 62.1 & 34.1 & 60.3 & 36.5 & 58.6 \\
\hline V1 & 30.6 & 19 & 29.4 & 13.8 & 32.9 & 13.8 & 30.6 & 13.8 \\
\hline V2 & 14.1 & 12 & 28.2 & 24.1 & 32.9 & 25.9 & 32.9 & 27.6 \\
\hline$P$ value $^{\mathrm{a}}$ & 0.23 & & 0.04 & & 0.004 & & 0.006 & \\
\hline
\end{tabular}

${ }^{\text {a }}$ Chi square test

${ }^{\mathrm{b}}$ Percentage between two age groups with every characteristic

Table 3 Multivariate Cox Proportional Hazard Ratio for factors associated with criteria of surgical success

\begin{tabular}{|c|c|c|c|c|}
\hline \multirow[b]{3}{*}{ Factor } & \multicolumn{4}{|c|}{ Criteria of surgical success } \\
\hline & \multicolumn{2}{|l|}{ Complete success } & \multicolumn{2}{|l|}{ Qualified success } \\
\hline & Hazard ratio $(95 \% \mathrm{CI})$ & $P$ value & Hazard ratio $(95 \% \mathrm{CI})$ & $P$ value \\
\hline Age & $1.02(1.01-1.03)$ & 0.001 & $1.00(0.99-1.01)$ & 0.53 \\
\hline Gender & + & & + & \\
\hline Family history of glaucoma & 0.00 & 0.95 & + & \\
\hline Diabetes mellitus & + & & + & \\
\hline Hypertension & $1.27(0.90-1.80)$ & 0.17 & $0.96(0.70-1.32)$ & 0.80 \\
\hline Type of glaucoma & & 0.06 & & 0.02 \\
\hline POAG & $1.52(0.92-2.52)$ & 0.10 & $1.74(1.07-2.82)$ & 0.02 \\
\hline PACG & $2.23(0.82-6.09)$ & 0.12 & $1.77(0.60-5.24)$ & 0.30 \\
\hline UG & $0.94(0.52-1.69)$ & 0.84 & $1.23(0.68-2.24)$ & 0.49 \\
\hline DG & $1.58(0.77-3.24)$ & 0.21 & $0.40(0.13-1.24)$ & 0.11 \\
\hline OSG & 1.00 & & 1.00 & \\
\hline Preoperative IOP & + & & $0.93(0.91-0.95)$ & $<0.001$ \\
\hline No. of anti-glaucoma medications ${ }^{\mathrm{a}}$ & & 0.001 & & 0.02 \\
\hline Preoperative cataract surgery & + & & $0.78(0.46-1.33)$ & 0.36 \\
\hline Phacotrabeculectomy & $0.66(0.32-1.37)$ & 0.26 & $0.61(0.36-1.05)$ & 0.08 \\
\hline Postoperative cataract surgery & + & & + & \\
\hline Visual field index (MD) & + & & + & \\
\hline
\end{tabular}

${ }^{a}$ number of preoperative anti-glaucoma medications

$C I$ Confidence interval, $P O A G$ Primary open-angle glaucoma, $P A C G$ Primary angle-closure glaucoma, $U G$ Uveitic glaucoma, $D G$ Developmental glaucoma, $O S G$ Other secondary glaucoma, $T L E$ Trabeculectomy; enumber of preoperative anti-glaucoma medications, $M D$ mean deviation of visual field indexes before trabeculectomy

+ characteristics did not have $P$ value $\leq 0.10$ in univariate analysis 
glaucoma $(P=0.03)$, number of preoperative antiglaucoma medications $(P<0.001)$ and phacotrabeculectomy $(P=0.06)$ (Supplementary Table 1$)$. In the multivariate analysis, the independent associations between complete success and age at surgery $(P=0.001)$ and number of preoperative anti-glaucoma medications $(P=$ 0.001) were maintained (Table 3).

\section{Qualified-success criteria}

In the univariate analysis, age at surgery $(P=0.002)$, the presence of systemic hypertension $(P=0.10)$, type of glaucoma $(P<0.001)$, number of preoperative antiglaucoma medications $(P=0.10)$, preoperative IOP $(P<0.001)$ and phacotrabeculectomy $(P=0.002)$ were associated with qualified success (Supplementary Table 1). In the multivariate analysis, type of glaucoma $(P=0.02)$, number of preoperative anti-glaucoma medications $(P=0.02)$ and preoperative IOP $(P<0.001)$ were associated with qualified success (Table 3 ).

\section{Discussion}

The main purpose of our study was to investigate whether age is associated with bleb morphology or success after trabeculectomy with MMC. Even in the pre-MMC era of filtration surgery, the success rate was known to be poorer in younger patients than in older [6]. In a series of trabeculectomies on patients under age 50, Gressel et al. [7] reported success rates (IOP $\leq 21 \mathrm{~mm} \mathrm{Hg}$ with or without medications or $22-25 \mathrm{~mm} \mathrm{Hg}$ without medications) of $35 \%$ for developmental glaucoma (DG) and $48 \%$ for secondary glaucoma (after a mean follow-up of 34 months and 32 months). Their success rate for patients aged 30-49 with POAG or primary angle-closure glaucoma (PACG) was $83 \%$ (25 of 30 eyes), which was considerably higher than for patients under 30 (44\%; 4 of 9 eyes). In the Advanced Glaucoma Intervention Study (AGIS) [8] and a retrospective study of trabeculectomy using MMC in pseudophakic eyes, younger age was associated with lower success, but in the studies of Fontana et al. [9] on phakic eyes undergoing trabeculectomy with MMC and Henry et al. [10] on phakic and pseudophakic eyes undergoing trabeculectomy with or without MMC, age did not influence the success rate. In our study, complete success $(\geq 30 \%$ IOP reduction without anti-glaucoma medications) and qualified success ( $\geq 30 \%$ IOP reduction with or without anti-glaucoma medications) showed significant differences between the two age groups. In our survival analysis, the rate of complete success at 48 months (4 years) in the older-age group ( $>60$ years) was $27.1 \%$, lower than that in the younger-age group ( $\leq 60$ years) $(41.7 \%)$. Moreover, only $67.9 \%$ of older- age eyes at 48 months ( 4 years) met the qualified-success criteria, compared with $75.6 \%$ of younger-age eyes.[10]: .

Our baseline characteristic data showed that there were significant post-trabeculectomy differences between the two groups in presence of hypertension, type of glaucoma, preoperative IOP and postoperative cataract surgery. To determine whether those factors affected the surgical success rates or not, Cox proportional hazard models for risk factors were run (Table 3 and Supplementary Table 1); the results revealed that the presence of hypertension affected the rates of complete success $(P=0.09$, hazard ratio (HR) $=0.87)$ and qualified success $(P=0.10, \mathrm{HR}=0.79)$ significantly by univariate analysis, but not by multivariate analysis.

The univariate analysis also showed that the type of glaucoma was a risk factor significantly associated with all of the success criteria. Among the types of glaucoma, POAG was associated with the qualified-success criteria $(P$ $=0.02, \mathrm{HR}=1.74$ ) by multivariate analysis. However, according to the Cox proportional hazard models, glaucoma type had no relation to the complete-success criteria in the multivariate analysis.

As there were more uveitic glaucomas and developmental glaucomas in the younger-age group, reanalysis was performed excluding those types of glaucoma. Age at surgery and number of preoperative anti-glaucoma medications were maintained as significant factors for complete success (Supplementary Tables 3 and 4).

Henry et al. [10] found lower preoperative IOP to be associated with trabeculectomy failure. Our findings showed that preoperative IOP was not associated with complete success but was, in fact, associated with qualified success. We also observed that the number of anti-glaucoma medications before trabeculectomy was associated with both complete success and qualified success. Moreover, the duration of medication use before trabeculectomy in the older-age group (51.9 \pm 40 months) was longer than in the younger-age group $(34.3 \pm 35.5),(P=0.006)$. Thus, there is a possibility that the longer duration of medication might have influenced the lower success in the older-age group. In our analysis of the cases that had undergone cataract surgery after trabeculectomy (range: 10 months -5 years), the Cox proportional hazard models showed no relation to any success criteria. This finding contradicted previous studies reporting post-trabeculectomy cataract-surgery associations with increased risk of bleb failure within the first 5 postoperative years $[11,12]$.

The results for phacotrabeculectomy were, in some previous findings, not as effective as trabeculectomy in reducing IOP $[13,14]$. We found, in our univariate analysis, that phacotrabeculectomy affected all of the success criteria; in our multivariate analysis however, there were no associations with any success criteria (Table 3 ). 
Bleb morphology is integral to long-term prediction of bleb function and IOP. Morphologic characteristics of successful filtration blebs, early signs of filtration failure and correlative IOP readings were noted by Shingleton [15], who established, based on that data, a categorization framework to identify the tell-tale patterns of failure. Also, various bleb grading systems, such as the Moorefield, the Indiana, and the Wuerzburg bleb classification score, have been introduced [16-18]. In our study, we used our own grading system, according to which all of the blebs were assessed by one glaucomatologist for bleb height, extent and vascularity. Favorable bleb development is associated with scarcity of vessels, large surface area and moderate elevation [15, 19]. The findings of Furrer et al. [16] and Thatte et al. [20] showed that the presence of vascularization is correlated with increased IOP, whereas Hirooka et al. [21]. observed that developing avascular and localized bleb after fornix-based trabeculectomy with MMC might be associated with increasing IOP. In our study, we found that there was a favorable correlation between avascularity and complete-success criteria $(P=0.04)$, as well as between prominent bleb and complete-success criteria $(P=0.03)$ (Supplementary Table 2). Also, we noted that avascularity $(66 \%)$ and prominent bleb $(65.7 \%)$ were characteristic of the younger-age group (Supplementary Figure 2). Vesti [22] and Thatte et al. [20] found a clear association between a diffuse bleb and a good IOP response. However, we observed that diffuse bleb was not correlated with complete success $(P=0.25)$. Our results showed that diffuse bleb $(55.9 \%)$ was more frequently found in the older-age group at 6 and 12 months $(P=0.004$ and $P=0.002$, respectively) (Table 2 and Supplementary Figure 2).

Poor success rates in young (<age 40) patients have been attributed in part to the thickness of Tenon's capsule and their more vigorous healing response [23, 24]. MMC has been shown to inhibit conjunctival/episcleral fibroblast proliferation, thus preventing scarring, and produces filtration blebs that are thinner, avascular, and more hypocellular with more atrophic stroma, thereby affording better surgical outcomes [25]. Philips [26] reported a 91\% 5-year-followup success rate for young patients (aged 15-40) who had undergone trabeculectomy with MMC, compared with just $73 \%$ for a group that had undergone trabeculectomy without MMC. On the other hand, Fontana et al. [9]. found that long-term (3-year follow-up) low IOP was achieved for only half of older ( $\geq$ age 40 ) patients who had undergone trabeculectomy with MMC. Indeed, in our younger-age group relative to the older-age group, we found more prominent blebs with a paucity of vascularity; accordingly, the younger-age group's long-term surgical outcomes were better than the older-age group's.

In conclusion, the younger-age group ( $\leq 60$ years) showed a significantly higher rate of long-term success than did the older-age group ( $>60$ years) after trabeculectomy with adjunctive MMC. Avascularity, prominence and localization were the characteristic bleb morphologies in the younger-age group. In addition, avascular and prominent blebs can provide for good filtering functionality.

\section{Summary}

\section{What was known before}

- Age is one of risk facters of trabeculectomy success.

- Younger individuals have worses success after trabeculectomy with adjunctive Mitomycine C (MMC).

- Blebs with scarcity of vessels, large surface area and moderate elevation were favorable filtering bleb.

\section{What this study adds}

- Long-term trabeculectomy success with adjunctive MMC in younger-age group was better than in olderage group.

- Good filtering functional bleb were avascular and prominent blebs.

Acknowledgements KHP had full access to the data in the study and takes responsibility for the integrity of the data and the accuracy of the data analysis. The authors alone are responsible for the content and writing of the paper. This research received no specific grant from any funding agency.

\section{Compliance with ethical standards}

Conflict of interest The authors declare that they have no conflict of interest.

\section{References}

1. Cairns JE. Trabeculectomy-preliminary report of a new method. Am J Ophthalmol. 1968;66:673-9.

2. Skuta GL, Parrish IIRK. Wound healing in glaucoma filtering surgery. Surv Ophthalmol. 1987;32:149-66.

3. Chen CW, Huang HT, Bair JS, Lee CC. Trabeculectomy with simultaneous topical application of mitomycine $\mathrm{C}$ in refractory glaucoma. J Ocul Pharmacol. 1990;6:175-82.

4. Palmer SS. Mitomycine as adjunct chemotherapy with trabeculectomy. Ophthalmology. 1991;98:317-21.

5. Skuta GL, Beeson CC, Higginbotham EJ, et al. Intraoperative mitomycine versus postoperative 5 -flouracil in high-risk glaucoma filtering surgery. Ophthalmolgy. 1992;99:438-44.

6. D'Ermo F, Bonomi L, Doro D. A critical analysis of the long-term results of trabeculectomy. Am J Ophthalmol. 1979;88:829-35.

7. Gressel MG, Heuer DK, Parrish RK II. Trabeculectomy in young patients. Ophthalmology. 1984;91:1242-6.

8. The AGIS Investigators. The Advanced Glaucoma Intervention Study (AGIS): 11. Risk factors for failure of trabeculectomy and argon laser trabeculoplasty. Am J Ophthalmol. 2002;134:481-98.

9. Fontana H, Nouri-Mahdavi K, Lumba J, Ralli M, Caprioli J. Trabeculectomy with mitomycin C: outcomes and risk factors for 
failure in phakic open-angle glaucoma. Ophthalmology. 2006;113:930-6.

10. Henry DJ, Jason FS, Patricia AT, Donna LG, Tara LL, Joan LJ, Harry AQ. Outcomes and bleb-related complications of trabeculectomy. Opththalmology. 2012;119:712-22

11. Patel HY, Danesh-Meyer HV. Incidence and management of cataract after glaucoma surgery. Curr Opin Ophthalmol. 2013;24:15-20.

12. Mathew RG, Murdoch IE. The silent enemy: a review of cataract in relation to glaucoma and trabeculectomy surgery. Br J Ophthalmol. 2011;95:1350-4.

13. Lochhead J, Casson RJ, Salmon JF. Long term effect on intraocular pressure of phacotrabeculectomy compared to trabeculectomy. Br J Ophthalmol. 2003;87:850-2.

14. Ogata-Iwao M, Inatani M, Takihara $Y$, Inoue $T$, Iwao K, Tanihara H. A prospective comparison between trabeculectomy with mitomycin C and phacotrabeculectomy with mitomycin C. Acta Ophthalmol. 2013;91:e500-e501.

15. Shingleton B. Management of the failing glaucoma filter. Ophthalmic Surg Lasers. 1996;27:445-51.

16. Furrer S, Menke MN, Funk J, Töteberg-Harms M. Evaluation of filtering blebs using the 'Wuerzburg bleb classification score' compared to clinical findings. BMC Ophthalmol. 2012;12:24.

17. Cantor LB, Mantravadi A, WuDunn D, Swamynathan K, Cortes A. Morphologic classification of filtering blebs after glaucoma filtration surgery: the Indiana Bleb Appearance Grading Scale. J Glaucoma. 2003;12:266-71.
18. Wells AP, Ashraff NN, Hall RC, Purdie G. Comparison of two clinical bleb grading systems. Ophthalmology. 2006;113:77-83.

19. Freedman SF, McCormick K, Cox TA. Mitomycin C-augumented trabeculectomy with postoperative wound modulation in pediatric glaucoma. J AAPOS. 1999;3:117-24.

20. Thatte S, Rana R, Gaur N. Appraisal of bleb using trio of intraocular pressure, morphology on slit lamp, and gonioscopy. Ophthalmol Eye Dis. 2016;8:41-48.

21. Hirooka K, Mizote M, Baba T, Takaghishi M, Shiraga F. Risk factors for developing avascular filtering bleb after fornix-based trabeculectomy with mitomycine C. J Glaucoma. 2009;18:301-4.

22. Vesti E. Filtering blebs: follow up of trabeculectomy. Ophthalmic Surg. 1993;24:249-55.

23. Beauchamp GR, Parks MM. Filtering surgery in children: barriers to success. Ophthalmology. 1979;86:170-80.

24. Khaw PT, Migdal CS. Current techniques in wound healing modulation in glaucoma surgery. Curr Opin Ophthalmol. 1996;7:24-33.

25. Mietz H, Arnold G, Kirchhof B, Diestelhorst M, Krieglstein GK. Histopathology of episcleral fibrosis after trabeculectomy with and without mitomycin C. Graefes Arch Clin Exp Ophthalmol. 1996;234:364-8.

26. Philip CJ, Thomas SD, Günter KK. Adjunctive mitomycin C in primary trabeculectomy in young adults: a long-term study of case-matched young patients. Graefe's Arch Clin Exp Ophthalmol. 1998;236:652-7. 